Case Report

\title{
Diagnosis of Cryptococcosis and Prevention of Cryptococcal Meningitis Using a Novel Point-of-Care Lateral Flow Assay
}

\begin{abstract}
Ashar Dhana
Department of Infectious Diseases, Helen Joseph Academic Hospital, 1 Perth Road, Westdene, Johannesburg 2092, South Africa

Correspondence should be addressed to Ashar Dhana; ashdha@hotmail.co.za

Received 26 August 2013; Accepted 8 October 2013

Academic Editor: Jacques F. Meis

Copyright (C) 2013 Ashar Dhana. This is an open access article distributed under the Creative Commons Attribution License, which permits unrestricted use, distribution, and reproduction in any medium, provided the original work is properly cited.

Despite access to antiretroviral therapy, mortality from cryptococcal meningitis (CM) is high among persons with advanced HIV infection in sub-Saharan Africa. Cryptococcal antigen (CrAg) is present several weeks to months before the onset of symptoms of meningitis and can be screened to prevent life threatening meningitis. Recently, the World Health Organisation recommended that a new rapid CrAg lateral flow "dipstick" assay (LFA) is to be used to screen HIV-infected persons with CD4 counts of less than 100 cells $/ \mu \mathrm{L}$. In this paper, we describe two cases of cryptococcosis with differing outcomes. In the first case, the new CrAg LFA was used as part of a screen and preemptive treatment strategy to prevent CM. In the second case, our patient had no access to the CrAg LFA and subsequently developed life threatening meningitis. To the best of our knowledge, this is the first case report of cryptococcosis diagnosed using this novel assay.
\end{abstract}

\section{Introduction}

Cryptococcosis is an infection caused by fungi that belong to the genus Cryptococcus. Two species in particularC. neoformans and C. gattii-cause nearly all cryptococcal infections in humans [1]. C. neoformans causes most cryptococcal infections in immunocompromised patients, while C. gattii causes disease in immunocompetent and, to a lesser extent, immunocompromised persons. Both species can infect any organ in the body, but most often they infect the lungs or invade the central nervous system, causing life threatening meningitis. Compared to C. neoformans, however, C. gattii is more commonly associated with isolated pulmonary infection, neurologic sequelae, and decreased in vitro susceptibility to common antifungal drugs $[2,3]$.

Cryptococcal meningitis (CM) is common in patients with advanced HIV infection, affecting an estimated 957,900 people per year [4]. Over $70 \%$ of these cases occur in subSaharan Africa, which bears a disproportionate burden of disease; $\mathrm{CM}$ is now the most common cause of meningitis in this setting [5]. Even in parts of the world with access to antiretroviral therapy (ART), mortality from CM is high with only $40-60 \%$ of patients being alive at 6 months $[4,6]$.
Current diagnostic tests for cryptococcosis include antigen tests and culture. Culture is the gold standard but is too insensitive [7]. Antigen tests are more commonly used to detect cryptococcal antigen $(\mathrm{CrAg})$ by either latex agglutination (LA) or enzyme immunoassay (EA). These tests are sensitive and specific but require technical expertise, a central reference laboratory, and special storage [8]. Recently, the CrAg lateral flow assay (LFA) - a new rapid "dipstick test"was developed to overcome problems with the diagnosis of $\mathrm{CM}$ in resource limited settings. Besides facilitating the early diagnosis of CM, the CrAg LFA shows great potential to prevent CM through targeted screening of HIV-infected individuals with advanced immunodeficiency [9]. We report two cases of cryptococcosis with contrasting outcomes and discuss the role of the new CrAg LFA in the diagnosis and prevention of $\mathrm{CM}$.

\section{Case Presentation 1}

A 38-year-old male was seen at his local clinic with a 6month history of loss of weight (approximately $10 \mathrm{~kg}$ ) and progressive loss of appetite. A rapid HIV test was performed 
and was positive. Additional blood tests confirmed a CD4 cell count of 2 cells $/ \mu \mathrm{L}$ and a positive CrAg LFA. Targeted screening of patients with CD4 cell counts of less than 100 cells $/ \mu \mathrm{L}$ for cryptococcal antigenemia was recently introduced into selected laboratories in South Africa as part of the National Strategic Plan on HIV, STIs, and TB. After a positive serum CrAg LFA test, the patient was referred to our hospital for a lumbar puncture to exclude CM. He reported no headache, neck stiffness, fever, visual impairment, or night sweats. Although he had a productive cough approximately 3 weeks ago, it had resolved with antibiotics prescribed by his local clinic. On examination, temporal wasting and generalized lymphadenopathy were present. Neurological examination revealed no neck stiffness, focal neurologic deficit, papilledema, or cranial nerve involvement. No cutaneous lesions or pulmonary signs were present. The remainder of the systemic examination was normal.

Laboratory blood tests showed a normocytic anaemia with haemoglobin of $11.8 \mathrm{~g} / \mathrm{dL}$. The cerebrospinal fluid (CSF) on admission was clear in appearance, and further analysis revealed lymphocytes of 1 cells $/ \mu \mathrm{L}$, neutrophils of 0 cells $/ \mu \mathrm{L}$, protein of $0.48 \mathrm{~g} / \mathrm{dL}$ (normal: $0.15-0.45 \mathrm{~g} / \mathrm{dL}$ ), and glucose of $3.2 \mathrm{mmol} / \mathrm{L}$ (normal: $2.8-4.4 \mathrm{mmol} / \mathrm{L}$ ). CSF Gram's stain, CrAg latex antigen (LA), and India ink were all negative. Xpert MTB/Rif assay of early morning sputum samples was negative for tuberculosis. A chest radiograph showed no abnormalities.

A diagnosis of disseminated cryptococcosis was made. Antifungal therapy was started with high dose fluconazole of $800 \mathrm{mg}$ daily according to the South African screen-andtreat algorithm for ART-naïve patients with a positive CrAg and negative lumbar puncture. Cotrimoxazole was also given as prophylaxis to prevent pneumonia from Pneumocystis jiroveci. The patient was discharged from hospital a day after his admission. At the 2-week follow-up visit, the fluconazole dose was reduced to $400 \mathrm{mg}$ daily and ART-with tenofovir, emtricitibine, and efavirenz-was initiated. At the 2-month visit, the fluconazole dose was further reduced to a maintenance dose of $200 \mathrm{mg}$ daily. So far he is doing well and reports no symptoms of meningitis.

\section{Case Presentation 2}

A 37-year-old female presented to the emergency department with a 1-week history of progressive neck stiffness, severe headache, fever, and loss of weight. She reported no other neurological symptoms. She had no medical history of TB but was diagnosed with HIV two years ago. At a visit to her local clinic about 3 weeks before this admission, laboratory tests showed a CD4 cell count of 76 cells $/ \mu \mathrm{L}$. She was subsequently started on antiretroviral therapywith tenofovir, emtricitabine, and efavirenz. Targeted screening for cryptococcal antigenemia was not yet offered by her clinic. The physical examination revealed tachycardia, pyrexia, conjunctival pallor, and generalized lymphadenopathy. Neck stiffness was present, but no confusion, focal neurologic deficit, papilledema, or cranial nerve involvement was evident on neurological examination. No pulmonary or dermatological changes were noted. The remainder of the systemic examination was otherwise normal.

The initial CSF opening pressure was raised $(28 \mathrm{~cm}$ $\mathrm{H}_{2} 0$ ). CSF was clear in appearance, and further analysis revealed lymphocytes of 34 cells $/ \mu \mathrm{L}$, neutrophils of 5 cells $/ \mu \mathrm{L}$, protein of $1.83 \mathrm{~g} / \mathrm{dL}$ (normal: $0.15-0.45 \mathrm{~g} / \mathrm{dL}$ ), and glucose of $1.9 \mathrm{mmol} / \mathrm{L}$ (normal: $2.8-4.4 \mathrm{mmol} / \mathrm{L}$ ). Gram's stain of the CSF was negative. The cryptococcal latex antigen test (CLAT) and India ink were, however, positive. CSF CrAg titres are not routinely performed in South Africa, as antifungal treatment is standardised and fungal burden at the time of diagnosis is invariably high. Other laboratory tests showed a positive peripheral blood CrAg LFA and a normocytic anaemia (haemoglobin: 9.2 g/L). Chest radiography was normal.

A diagnosis was made of cryptococcal meningitis with raised intracranial pressure and unmasking HIV-associated IRIS. ART was continued in the ward, and antifungal therapy was started with amphotericin B (1 mg/kg/day IV) and high dose fluconazole of $800 \mathrm{mg}$ daily. Fluconazole is used in South Africa, as flucytosine is presently unavailable. Intravenous antimicrobial therapy with ceftriaxone was also started. To avoid nephrotoxicity and electrolyte abnormalities, she was prehydrated and given potassium and magnesium supplements. No nephrotoxicity or worsening of anaemia was noted during admission. Therapeutic lumbar puncture was performed three times a week to relieve symptoms of raised intracranial pressure, and paracetamol was given for further analgesia. Corticosteroids were also added to the regimen. By hospital day 14, her symptoms had improved greatly. Intravenous amphotericin was stopped and the dose of fluconazole was changed to $400 \mathrm{mg}$ daily. She was discharged from hospital 3 weeks after admission. At her 2-month follow-up visit, she was maintained on fluconazole of $200 \mathrm{mg}$ daily as secondary prophylaxis. She is currently well and reports no recurrence of her symptoms.

\section{Discussion}

These cases demonstrate the utility of the new CrAg LFA assay in the diagnosis of cryptococcosis and prevention of CM.

A large proportion of $\mathrm{CM}$ is preventable for several reasons. First, $\mathrm{CrAg}$ is present in peripheral blood for an average of 22 days before the onset of $\mathrm{CM}$, and in $10 \%$ of cases it may be present for more than 100 days before the onset of meningitis [10]. As seen with our first patient, this asymptomatic phase is important for identifying those at risk of developing clinical disease at a later stage. Second, in HIVinfected individuals with CD 4 counts of less than 100 cells $/ \mu \mathrm{L}$, up to $12 \%$ have been shown to be CrAg positive [8]. One retrospective study showed that almost $30 \%$ of these $\mathrm{CrAg}$ positive patients will go on to develop CM [11]. Finally, in one study, over $70 \%$ of patients who were diagnosed with CM already knew their HIV status. Moreover, many of these patients were recently tested for HIV (within the last 4 months), but the diagnosis of cryptococcosis was missed [12]. As a result, it has become clear that screening for $\mathrm{CrAg}$ in 


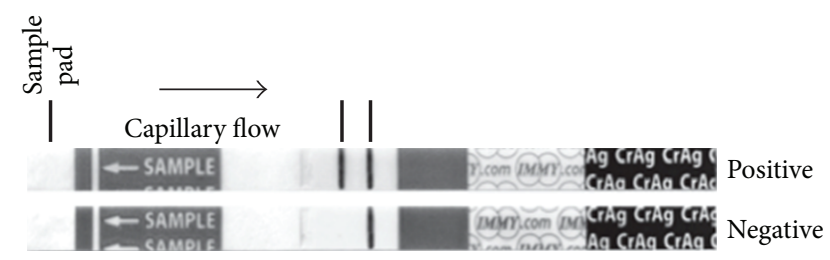

FIgURE 1: The cryptococcal antigen lateral-flow assay is read in the same way as that of a urine pregnancy dipstick test. The presence of two visible bands indicates a positive result.

advanced immunodeficiency plays an important role in any ART programme.

Recently, the newly developed CrAg LFA (Figure 1) has shown promise as a diagnostic and screening tool for cryptococcosis, meeting most of the WHO criteria for an ideal point-of-care test [13]. The assay provides a result in less than 10 minutes and requires no special storage or prior laboratory expertise. Of four prospective and retrospective studies, the CrAg LFA showed excellent diagnostic accuracy when compared to culture and CrAg LA or EA $[9,14-16]$. On CSF and peripheral blood samples, sensitivity ranged from $96 \%$ to $100 \%$ and specificity was $92 \%$ to $100 \%$. In these studies, agreement and correlation between the CrAg LFA and other diagnostic assays were also shown to be very high. The assay also shows acceptable sensitivity (70-98\%) with urine samples, which are easier to collect and pose minimal biohazard risk $[9,14,15]$. Furthermore, at a cost of $\$ 2.50$, the CrAg LFA is less than half the cost of the more common assays [15]. In one prospective study in Uganda, screening with the CrAg LFA was found to be more cost-effective than a prophylactic course of antifungal treatment in HIV-infected patients with advanced immunodeficiency [17].

Early diagnosis of cryptococcosis-either subclinical or clinical-is a crucial aspect of infectious disease control, especially in resource limited settings. The new CrAg LFA shows potential as a point-of-care test and may substantially reduce the global burden of cryptococcal meningitis. Given the high morbidity and mortality of CM, further studies are needed on how to best implement this novel test into existing ART programmes and diagnostic algorithms.

\section{Conflict of Interests}

The author declares that there is no conflict of interests regarding the publication of this paper.

\section{Acknowledgment}

The author would like to thank the National Health Laboratory Service for performing the CrAg LFA on samples obtained from both of these patients.

\section{References}

[1] S. Antinori, "New insights into HIV/AIDS-associated cryptococcosis," ISRN AIDS, vol. 2013, Article ID 471363, 22 pages, 2013.
[2] K. Datta, K. H. Bartlett, R. Baer et al., "Spread of Cryptococcus gattii into Pacific Northwest Region of the United States," Emerging Infectious Diseases, vol. 15, no. 8, pp. 1185-1191, 2009.

[3] Z. U. Khan, H. S. Randhawa, T. Kowshik, A. Chowdhary, and R. Chandy, "Antifungal susceptibility of Cryptococcus neoformans and Cryptococcus gattii isolates from decayed wood of trunk hollows of Ficus religiosa and Syzygium cumini trees in NorthWestern India," Journal of Antimicrobial Chemotherapy, vol. 60, no. 2, pp. 312-316, 2007.

[4] B. J. Park, K. A. Wannemuehler, B. J. Marston, N. Govender, P. G. Pappas, and T. M. Chiller, "Estimation of the current global burden of cryptococcal meningitis among persons living with HIV/AIDS," AIDS, vol. 23, no. 4, pp. 525-530, 2009.

[5] J. N. Jarvis, G. Meintjes, A. Williams, Y. Brown, T. Crede, and T. S. Harrison, "Adult meningitis in a setting of high HIV and TB prevalence: Findings from 4961 suspected cases," BMC Infectious Diseases, vol. 10, article 67, 2010.

[6] D. R. Boulware, D. B. Meya, T. L. Bergemann et al., "Clinical features and serum biomarkers in HIV immune reconstitution inflammatory syndrome after cryptococcal meningitis: a prospective cohort study," PLoS Medicine, vol. 7, no. 12, Article ID e1000384, 2010.

[7] D. C. Tanner, M. P. Weinstein, B. Fedorciw, K. L. Joho, J. J. Thorpe, and L. B. Reller, "Comparison of commercial kits for detection of cryptococcal antigen," Journal of Clinical Microbiology, vol. 32, no. 7, pp. 1680-1684, 1994.

[8] R. Rajasingham, D. B. Meya, and D. R. Boulware, "Integrating cryptococcal antigen screening and pre-emptive treatment into routine HIV care," Journal of Acquired Immune Deficiency Syndromes, vol. 59, no. 5, pp. e85-e91, 2012.

[9] J. N. Jarvis, A. Percival, S. Bauman et al., "Evaluation of a novel point-of-care cryptococcal antigen test on serum, plasma, and urine from patients with HIV-associated cryptococcal meningitis," Clinical Infectious Diseases, vol. 53, no. 10, pp. 10191023, 2011.

[10] N. French, K. Gray, C. Watera et al., "Cryptococcal infection in a cohort of HIV-1-infected Ugandan adults," AIDS, vol. 16, no. 7, pp. 1031-1038, 2002.

[11] J. N. Jarvis, S. D. Lawn, M. Vogt, N. Bangani, R. Wood, and T. S. Harrison, "Screening for cryptococcal antigenemia in patients accessing an antiretroviral treatment program in South Africa," Clinical Infectious Diseases, vol. 48, no. 7, pp. 856-862, 2009.

[12] J. N. Jarvis, G. Meintjes, R. Wood, and T. S. Harrison, “Testing but not treating: missed opportunities and lost lives in the South African antiretroviral therapy programme," AIDS, vol. 24, no. 8, pp. 1233-1235, 2010.

[13] R. W. Peeling and D. Mabey, "Point-of-care tests for diagnosing infections in the developing world," Clinical Microbiology and Infection, vol. 16, no. 8, pp. 1062-1069, 2010.

[14] B. J. McMullan, C. Halliday, T. C. Sorrell et al., "Clinical utility of the cryptococcal antigen lateral flow assay in a diagnostic mycology laboratory," PLoS ONE, vol. 7, no. 11, Article ID e49541, 2012.

[15] M. D. Lindsley, N. Mekha, H. C. Baggett et al., "Evaluation of a newly developed lateral flow immunoassay for the diagnosis of cryptococcosis," Clinical Infectious Diseases, vol. 53, no. 4, pp. 321-325, 2011.

[16] J. Hansen, E. S. Slechta, M. A. Gates-Hollingsworth et al., "Large-scale evaluation of the immuno-mycologics lateral flow and enzyme-linked immunoassays for detection of cryptococcal antigen in serum and cerebrospinal fluid," Clinical and Vaccine Immunology, vol. 20, no. 1, pp. 52-55, 2013. 
[17] D. B. Meya, Y. C. Manabe, B. Castelnuovo et al., "Cost-effectiveness of serum cryptococcal antigen screening to prevent deaths among HIV-infected persons with a CD4+ cell count $\leq 100$ cells $/ \mu \mathrm{L}$ who start HIV therapy in resource-limited settings," Clinical Infectious Diseases, vol. 51, no. 4, pp. 448-455, 2010. 


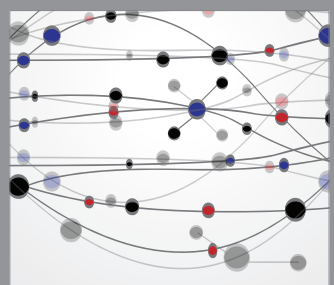

The Scientific World Journal
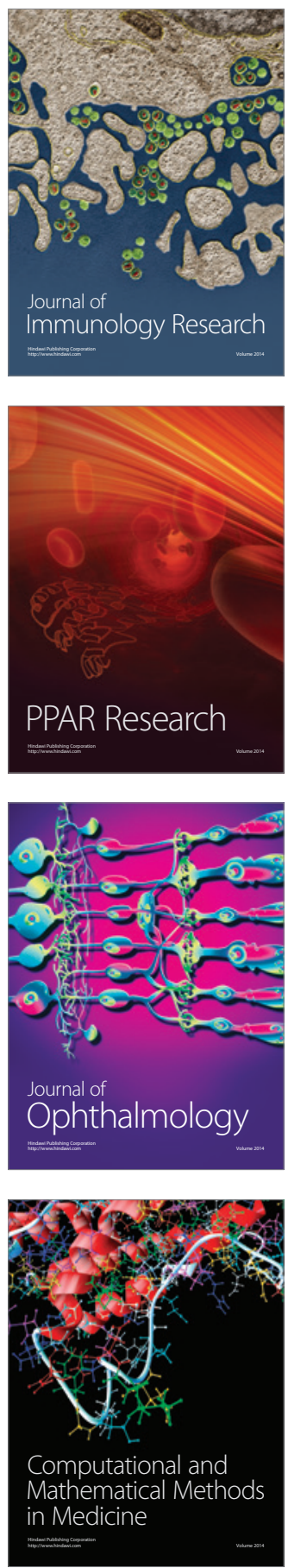

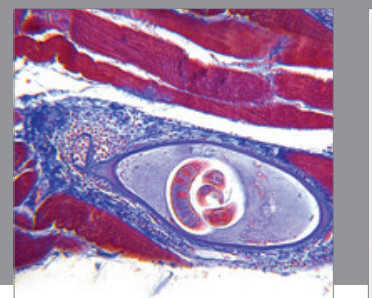

Gastroenterology

Research and Practice
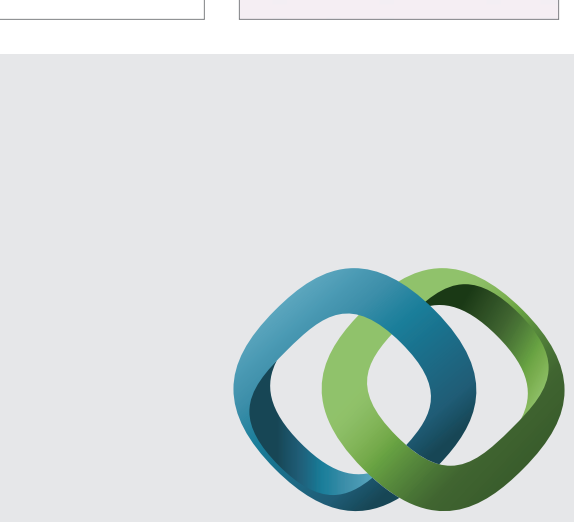

\section{Hindawi}

Submit your manuscripts at

http://www.hindawi.com
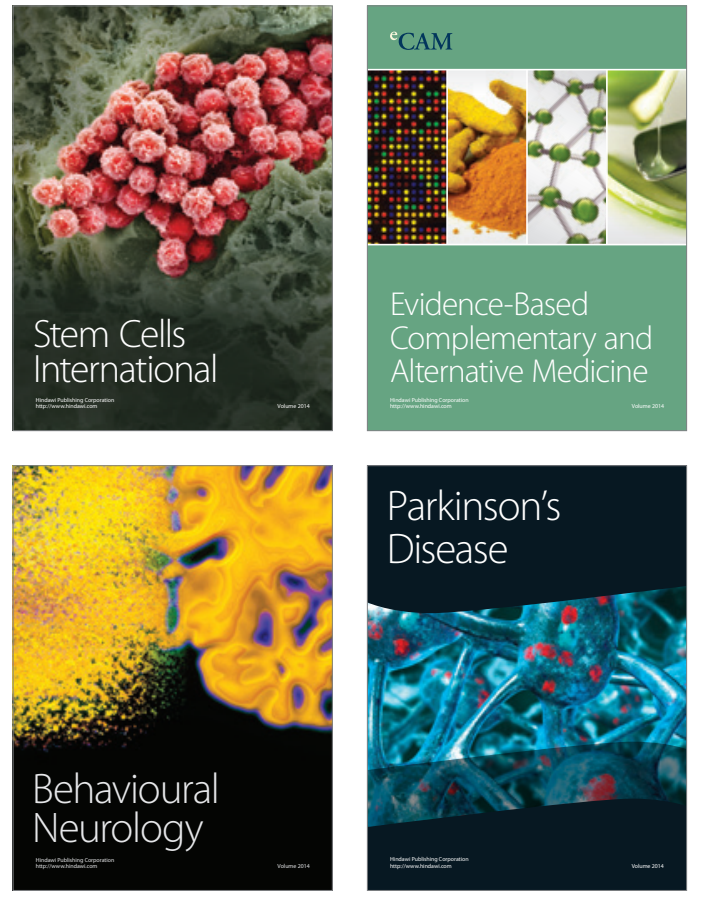
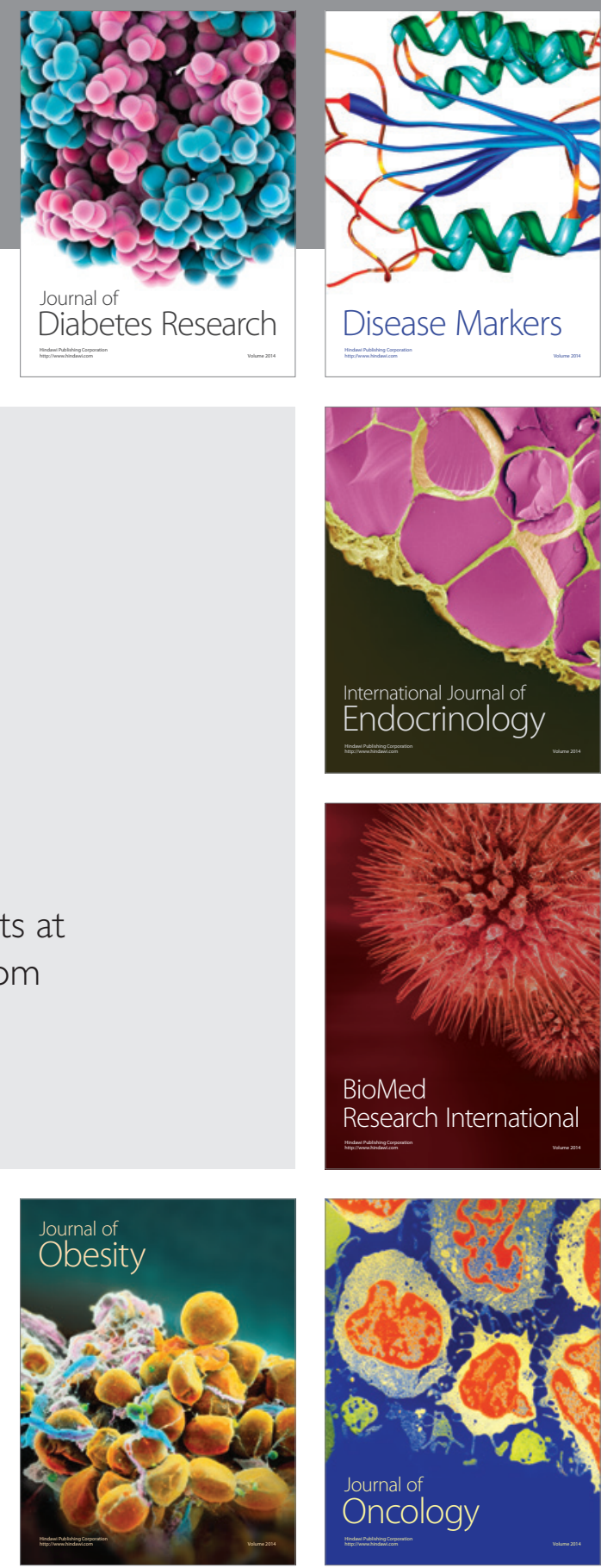

Disease Markers
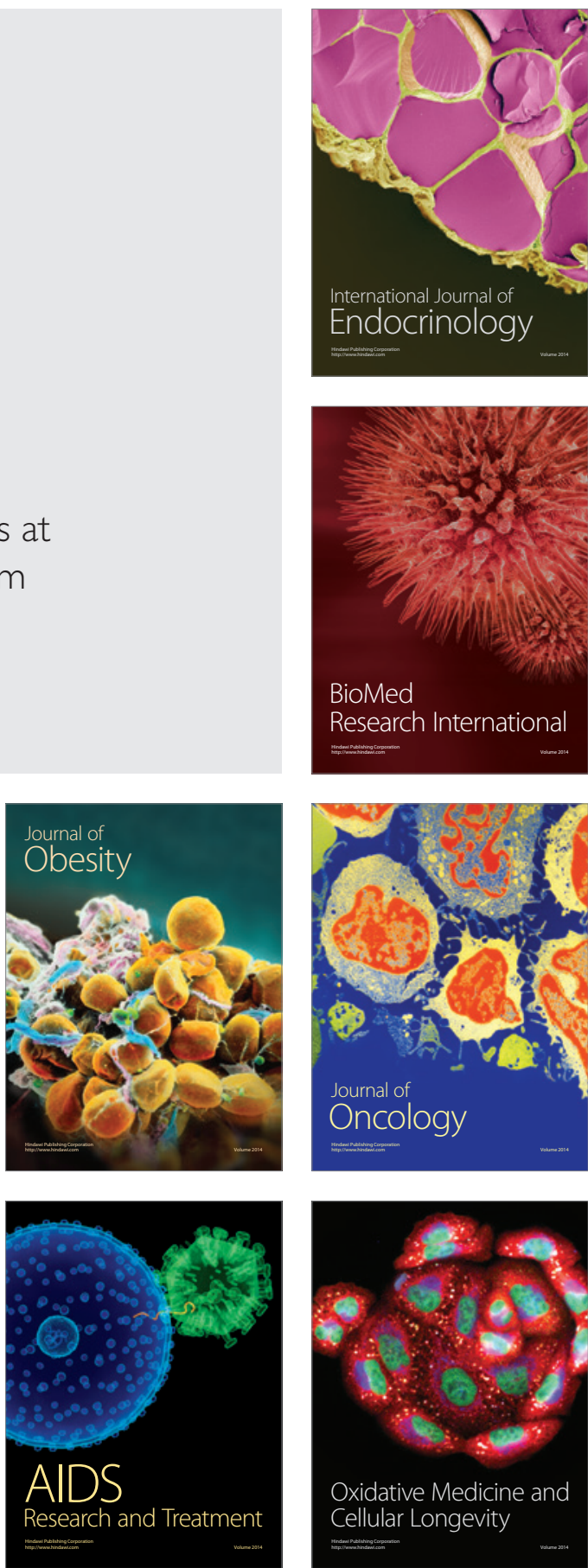